\title{
Absence of Ballistic Motion
}

\section{Barry Simon*}

Division of Physics, Mathematics, and Astronomy, California Institute of Technology,

Pasadena, CA 91125, USA

Received February 20, 1990

Abstract. For large classes of Schrödinger operators and Jacobi matrices we prove that if $h$ has only one point spectrum then for $\phi_{0}$ of compact support

$$
\lim _{t \rightarrow \infty} t^{-2}\left\|x e^{-i t h} \phi_{0}\right\|^{2}=0 \text {. }
$$

\section{Introduction}

Consider a free Schrödinger particle. Then the Heisenberg position operators obeys

$$
x(t)=x+t p
$$

since $p$ is a constant of the motion. Thus $|x(t)|$ grows linearly in $t$, indeed for any $\phi \in \mathscr{S}\left(\mathbb{R}^{n}\right)$ :

$$
\lim \left(\phi, x(t)^{2} \phi\right) / t^{2}=\left(\phi, p^{2} \phi\right)>0 .
$$

This paper had its root in a question of Joel Lebowitz asking if such ballistic motion didn't have its roots in absolutely continuous spectrum. Alas, while it is likely that Joel is correct, I have been able to obtain only partial results. Here I will prove that for Hamiltonians with pure point spectrum (think of the random case [1]), we have that for a dense set of initial $\phi$ that $\left(\phi, x(t)^{2} \phi\right) / t^{2} \rightarrow 0$. Unfortunately, I have nothing to say in the singular continuous case.

For background note that it is a result of Radin-Simon [2] that when $\phi$ is in $C_{0}^{\infty},\left(\phi, x(t)^{2} / t^{2}\right.$ is bounded at infinity in great generality.

I'd like to thank Joel for his question. I'd like to thank Tom Spencer, both for several useful conversations and for the hospitality of the Institute for Advanced Studies, where this work was done. The support of a Guggenheim fellowship during the period in question is also acknowledged with thanks.

* Research partially supported by NSF grant number DMS-8801918 


\section{The Discrete Case}

On $l^{2}\left(\mathbb{Z}^{0}\right)$, let $h_{0}$ be defined by

$$
\left(h_{0}\right)(n)=\sum_{|m-n|=1} u(m) .
$$

If $v$ is an arbitrary real valued function and also the operator of multiplication by $v$ on $D(v)=\left\{\left.u\left|\Sigma(|v(n)|+1)^{2}\right| u(n)\right|^{2}<\infty\right\}$, then $h=h_{0}+v$ is self-adjoint on $D(v)$ since $h_{0}$ is bounded.

Define

$$
(\mathbf{x} u)(n)=\mathbf{n} u(\mathbf{n})
$$

and $\mathbf{p}=i\left[h_{0}, \mathbf{x}\right]$ formally, explicitly

$$
(\mathbf{p} u)(n)=-\sum_{|j|=1} i j u(n+\mathbf{j}) .
$$

Then $\mathbf{p}$ is bounded. Moreover, we claim that if

$$
\mathbf{x}(t)=e^{i t H} \mathbf{x} e^{-i t H}, \quad \mathbf{p}(t)=e^{i t H} \mathbf{p} e^{-i t H},
$$

then

$$
\mathbf{x}(t)=\mathbf{x}+\int_{0}^{t} \mathbf{p}(s) d s
$$

as forms on $D(x)$. For it is easy to see that $\mathbf{x}(0)$ is bounded and equal to $p$. Thus, we have, since $p$ is bounded:

Lemma 1.1. For $\phi \in D(\mathbf{x})$ :

$$
\lim _{t \rightarrow \infty} \frac{1}{t^{2}}\left(\phi,|\mathbf{x}(t)|^{2} \phi\right)=\lim _{t \rightarrow \infty} \frac{1}{t^{2}} \int_{0}^{t} d s \int_{0}^{t} d u(\phi, \mathbf{p}(u) \cdot \mathbf{p}(s) \phi) .
$$

With this we prove:

Theorem 1.2. Suppose that $h$ has only point spectrum. Then for $\phi \in D(\mathbf{x})$.

$$
\lim _{t \rightarrow \infty}\left(\phi,|\mathbf{x}(t)|^{2} \phi\right) / t^{2}=0 .
$$

Proof. We will show the right-hand side of (1) goes to 0 for all $\phi$. The integrand in (1) is uniformly bounded, so it suffices to prove the result for a dense set of $\phi$, say a finite sum of eigenfunctions of $h$. Let $\phi_{n}$ be a complete set of eigenfunctions of $h$ :

$$
h \phi_{n}=e_{n} \phi_{n} .
$$

Thus we need only show that for all $n, m$ :

$$
\frac{1}{t^{2}} \int_{0}^{t} d s \int_{0}^{t} d u\left(\phi_{n}, \mathbf{p}(u) \cdot \mathbf{p}(s) \phi_{n}\right) \rightarrow 0 \text {. }
$$


Let $\mathbf{p}_{n k}=\left(\phi_{n}, \mathbf{p} \phi_{k}\right)$ and

$$
f_{n, m, k}(t)=t^{-2} \frac{1}{t^{2}} \int_{0}^{t} d s \int_{0}^{t} d u e^{-i u\left(E_{k}-E_{n}\right)} e^{-i s\left(E_{m}-E_{k}\right)} .
$$

so

$$
\text { left-hand side of }(2)=\sum_{k} \mathbf{p}_{n k} \cdot \mathbf{p}_{k m} f_{n, m, k}(t)
$$

Next note that $|f| \leq 1$ and

$$
\sum_{k}\left|\mathbf{p}_{n k} \cdot \mathbf{p}_{k m}\right| \leq\left(\sum_{k} \mid \mathbf{p}_{k m}^{2}\right)^{1 / 2}\left(\sum_{k}\left|\mathbf{p}_{k m}\right|^{2}\right)^{1 / 2}=\left\|p \phi_{n}\right\|\left\|p \phi_{m}\right\| .
$$

Thus by the dominated convergence theorem, it suffices to show that for each $n, m, k$ either $\mathbf{p}_{n k} \cdot \mathbf{p}_{k m}=0$ or $f_{n, m, k}(t) \rightarrow 0$ as $t \rightarrow \infty$. The integral determining $f$ is easy to do and one sees that $f(t) \rightarrow 0$ unless $E_{n}=E_{k}=E_{m}$. Thus the theorem follows from the virial theorem (Lemma 2.3) below.

Lemma 2.3. If $E_{n}=E_{k}$, then $\mathbf{p}_{n k}=0$.

Proof. Define $\mathbf{x}_{M}$ by

$$
\begin{aligned}
\left(\mathbf{x}_{M}\right)_{i} & =M & x_{i} & \geq M \\
& =x_{i} & \left|x_{i}\right| & \leq M \\
& =-M & x_{i} & \leq-M
\end{aligned}
$$

and $\mathbf{p}_{M}=i\left[h_{0}, \mathbf{x}_{M}\right]$. Then by a direct calculation

$$
s-\lim _{M \rightarrow \infty} \mathbf{p}_{M}=\mathbf{p},
$$

so it suffices that

$$
\left(\phi_{n}, \mathbf{p}_{M} \phi_{m}\right)=0 \text {. }
$$

Since $\mathbf{x}_{M}$ is bounded, this follows by expanding the commutator.

\section{The Continuum Case}

Theorem 3.1. Let $V$ be a multiplication operator on $L^{2}\left(\mathbb{R}^{n}\right)$ so that $H_{0}+V \equiv$ $-\Delta+V$ is bounded below on $Q\left(H_{0}\right) \cap Q(V)$ and let $H=H_{0}+V$ be the form closure. Suppose $Q(H) \subset Q\left(H_{0}\right)$. (Equivalently there is a form bound $H_{0} \leq c(H+d)$.) Let $\phi \in D(\mathbf{x}) \cap Q(H)$. Suppose that $H$ has only point spectrum. Then

$$
\lim _{t \rightarrow \infty}\left(\phi,|\mathbf{x}(t)|^{2} \phi\right) / t^{2}=0 .
$$

Proof. Except for technicalities, the same as Theorem 2.2. By Radin-Simon [2], $D(\mathbf{x}) \cap Q(H)$ is left invariant by $e^{i t H}$ and $\mathbf{x}(t)=\mathbf{x}+2 \int_{0}^{t} \mathbf{p}(s) d s$. As in Sect. 2 , it suffices to show for $\phi \in Q(H)$,

$$
\frac{1}{t^{2}} \int_{0}^{t} d s \int_{0}^{t} d u(\phi, \mathbf{p}(s) \cdot \mathbf{p}(u) \phi) \rightarrow 0 \text {. }
$$


Since $\mathbf{p}(s)(H+i)^{-1 / 2}$ is uniformly bounded, we need only show this for finite sums of eigenfunctions.

As in the proof of Lemma 2.3, we define $\mathbf{x}_{N}$ and $\mathbf{p}_{N}$ but with a slightly different formula. Pick $f(x), C^{\infty}$ on $\mathbb{R}$ so $f^{\prime} \geq 0$ and

$$
f(x)= \pm 1 \text { for } \pm x \geq 1=x \text { for }|x| \leq 1 / 2
$$

and define $x_{N}=N f(x / N)$ and $p_{N}=\frac{i}{2}\left[H_{0}, x_{N}\right] . \mathbf{x}_{N}$ is bounded but $\mathbf{p}_{N}$ is not. However for $\phi \in Q\left(H_{0}\right)$ we have $\left\|\left(\mathbf{p}_{N}-\mathbf{p}\right) \phi\right\| \rightarrow 0$ and so the argument in Lemma 2.3 extends.

\section{References}

1. Cycon, H., Froese, R., Kirsch, W., Simon, B.: Schrödinger Operators. Berlin, Heidelberg, New York: Springer 1987

2. Radin, C., Simon, B.: Invariant domains for the time-dependent Schrödinger equation. J. Diff. Eq. 29, 289-296 (1978)

Communicated by T. Spencer 